\title{
Stimulation of the Posterior Cingulate Cortex Impairs Episodic Memory Encoding
}

\author{
Vaidehi S. Natu, ${ }^{1}$ Jui-Jui Lin, ${ }^{1}$ Alexis Burks, ${ }^{1}$ Akshay Arora, ${ }^{1}{ }^{\oplus}$ Michael D. Rugg, ${ }^{2}$ and Bradley Lega ${ }^{1}$ \\ ${ }^{1}$ Department of Neurological Surgery, University of Texas Southwestern Medical Center, Dallas, Texas 75390, and ${ }^{2}$ Center for Vital Longevity, University of \\ Texas at Dallas, Texas 75080
}

\begin{abstract}
Neuroimaging experiments implicate the posterior cingulate cortex (PCC) in episodic memory processing, making it a potential target for responsive neuromodulation strategies outside of the hippocampal network. However, causal evidence for the role that PCC plays in memory encoding is lacking. In human female and male participants $(N=17)$ undergoing seizure mapping, we investigated functional properties of the PCC using deep brain stimulation (DBS) and stereotactic electroencephalography. We used a verbal free recall paradigm in which the PCC was stimulated during presentation of half of the study lists, whereas no stimulation was applied during presentation of the remaining lists. We investigated whether stimulation affected memory and modulated hippocampal activity. Results revealed four main findings. First, stimulation during episodic memory encoding impaired subsequent free recall, predominantly for items presented early in the study lists. Second, PCC stimulation increased hippocampal gamma-band power. Third, stimulation-induced hippocampal gamma power predicted the magnitude of memory impairment. Fourth, functional connectivity between the hippocampus and PCC predicted the strength of the stimulation effect on memory. Our findings offer causal evidence implicating the PCC in episodic memory encoding. Importantly, the results indicate that stimulation targeted outside of the temporal lobe can modulate hippocampal activity and impact behavior. Furthermore, measures of connectivity between brain regions within a functional network can be informative in predicting behavioral effects of stimulation. Our findings have significant implications for developing therapies to treat memory disorders and cognitive impairment using DBS.
\end{abstract}

Key words: deep brain stimulation; episodic memory; functional connectivity; hippocampus; posterior cingulate cortex; stereotactic electroencephalography

\section{Significance Statement}

Cognitive impairment and memory loss are critical public health challenges. Deep brain stimulation (DBS) is a promising tool for developing strategies to ameliorate memory disorders by targeting brain regions involved in mnemonic processing. Using DBS, our study sheds light on the lesser-known role of the posterior cingulate cortex (PCC) in memory encoding. Stimulating the PCC during encoding impairs subsequent recall memory. The degree of impairment is predicted by stimulation-induced hippocampal gamma oscillations and functional connectivity between PCC and hippocampus. Our findings provide the first causal evidence implicating PCC in memory encoding and highlight the PCC as a favorable target for neuromodulation strategies using $a$ priori connectivity measures to predict stimulation effects. This has significant implications for developing therapies for memory diseases.

\section{Introduction}

Deep brain stimulation (DBS) is a powerful tool used clinically to target dysregulated neural circuits and to treat neurological dis-

Received Feb. 19, 2019; revised May 29, 2019; accepted July 10, 2019.

Author contributions: V.S.N. and B.L. designed research; V.S.N., J.-J.L., A.B., A.A., and B.L. performed research; V.S.N. and J.-J.L. analyzed data; V.S.N. and B.L. wrote the paper; M.D.R. edited the paper.

This work was funded by the National Institute of Neurological Disorders and Stroke-National Institutes of Health (Grant R01 NS107357-02 to B.L.).

The authors declare no competing financial interests.

Correspondence should be addressed to Vaidehi S. Natu at vaidehi.natu@utsouthwestern.edu. orders including Parkinson's disease, essential tremor, psychiatric illnesses, and epilepsy (Limousin et al., 1998; Perlmutter and Mink, 2006; Kringelbach et al., 2007; Ressler and Mayberg, 2007; Bronstein et al., 2011). Several studies have applied DBS to patients undergoing treatment for epilepsy for understanding the effects of stimulation on human memory (Coleshill et al., 2004; Lacruz et al., 2010; Suthana et al., 2012; Fell et al., 2013; Jacobs et al., 2016; Ezzyat et al., 2017, 2018; Goyal et al., 2018; Inman et al., 


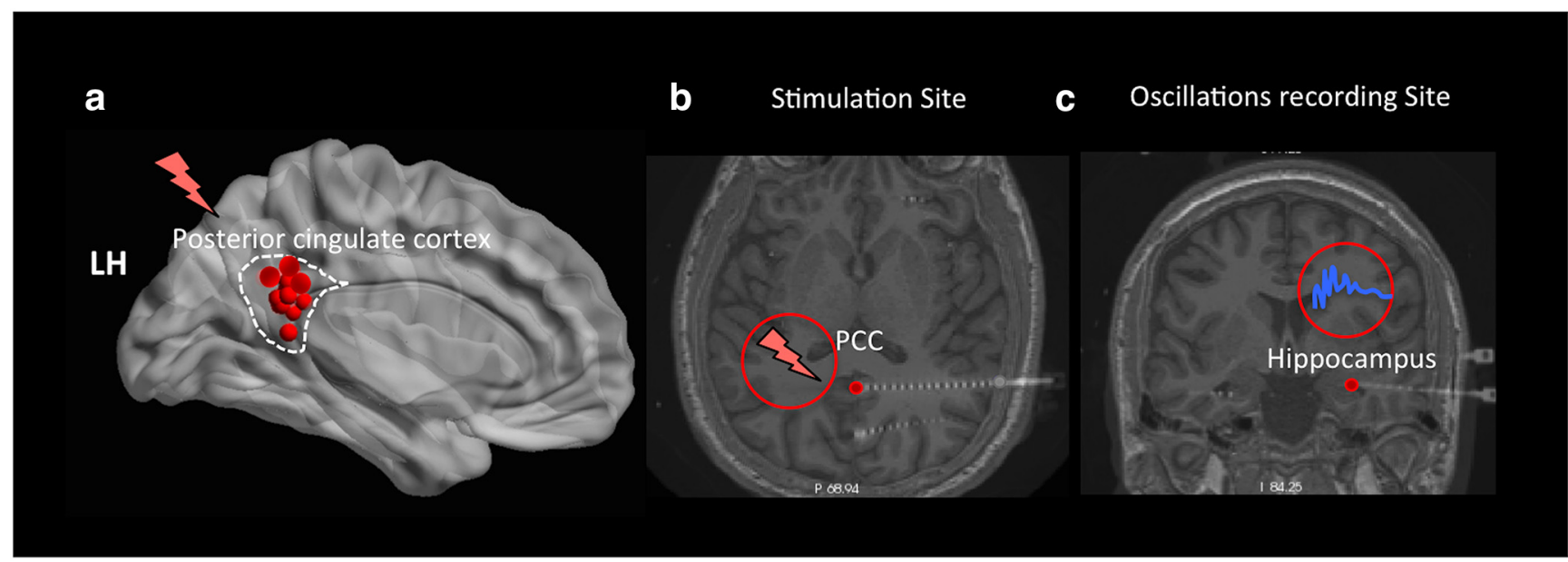

Figure 1. Electrode placement during a DBS paradigm. $\boldsymbol{a}$, A summary figure showing the left medial view of the Talairach brain with the locations of stimulated electrodes (red dots) across 16 of the 17 participants who had their stimulation sites in the left PCC. Red lightning bar represents stimulated sites. $\boldsymbol{b}, \boldsymbol{c}$, Transverse and coronal slices of the sample participant's brain showing the deep insertion of stereo electrodes in the PCC and hippocampus, respectively. DBS was applied to the PCC during the encoding phase in a free recall experiment and neural oscillations were recorded simultaneously from the hippocampus.

2018; Kucewicz et al., 2018). Electrical stimulation applied to core memory regions including the hippocampus and entorhinal cortex impairs memory (Jacobs et al., 2016; Goyal et al., 2018; with some exceptions showing memory enhancement, see Suthana et al., 2012; Suthana and Fried, 2014). These findings indicate the causal importance of cortical structures and implicate DBS as an ideal method for developing neuromodulation strategies to treat memory disorders (Johnson et al., 2013).

The posterior cingulate cortex (PCC) is a deep cortical region that supports episodic memory processing. The majority of the evidence on PCC's role in memory processing comes from noninvasive neuroimaging studies showing reduced neural activation for successfully remembered than forgotten items (negative subsequent memory effect, SME) during memory encoding (Otten and Rugg, 2001; Daselaar et al., 2004; Uncapher et al., 2006; Miller et al., 2008; Duverne et al., 2009; Huijbers et al., 2011). The PCC also exhibits increased activation during successful episodic retrieval (Maddock et al., 2001; Cabeza et al., 2004; Wagner et al., 2005; Huijbers et al., 2011; Sestieri et al., 2011; Rugg and Vilberg, 2013). Additionally, evidence of PCC's dense anatomical connections with medial temporal cortex and memory impairments associated with reduced metabolism in the PCC and abnormal functional connectivity between PCC and hippocampus (Minoshima et al., 1997; Greicius et al., 2004; Zhou et al., 2008; De Vogelaere et al., 2012; Leech and Sharp, 2014; Papma et al., 2017) make the PCC an excellent neuromodulation target for cognitive enhancement. Despite fMRI evidence, progress on PCC's role in memory encoding using electroencephalography (EEG) remains limited as PCC is buried deep inside the medial surface of the brain. Recent intracranial EEG (iEEG) work using grid electrodes reported that the posterior medial cortex (PMC), including the PCC, shows increase in high gamma-band activity in the PMC during memory retrieval (Foster et al., 2012, 2013, 2015; Fox et al., 2018), providing evidence for PMC's role in memory cognition. Here, we used DBS and iEEG to examine the role of PCC in episodic memory encoding and to determine whether PCC could be used as a target region for memory enhancement.

Based on prior fMRI findings (Otten and Rugg, 2001; Daselaar et al., 2004; Duverne et al., 2009) that episodic memory encoding is facilitated when neural activity in the PCC is attenuated, we reasoned that high-frequency stimulation delivered to PCC during encoding might enhance memory. To test this prediction, we stimulated the PCC (Fig. 1a) using robotically placed stereotactic EEG (stereo-EEG) electrodes implanted for seizure mapping (Lega et al., 2017; Lin et al., 2017). The stereo-EEG technique allows precise targeting of deep brain regions (Fig. 1b) and simultaneous acquisition of oscillatory recordings from neighboring regions such as the hippocampus (Fig. 1c). We used a free recall memory task in which participants memorized a list of sequentially presented words during an encoding period and then, after a brief distraction period, attempted to freely recall the words. PCC was stimulated during the entire encoding period for half of the study lists; no stimulation was applied during remainder of the lists. We examined if: (1) stimulation induced memory enhancement or impairment, (2) stimulation induced modulations in hippocampal oscillations, and (3) there was a relationship between behavioral and neural effects of stimulation.

\section{Materials and Methods}

Participants. We collected data from 17 participants (ages 19-63, 9 female) implanted with stereo-EEG electrodes and undergoing treatment for epilepsy. Patients were recruited from the epilepsy surgery practice at the University of Texas, Southwestern Medical Center. Only patients who had intracranial electrodes placed within the posterior cingulate and completed all trials in the experiment, were included in the study. All participants had normal or corrected-to-normal vision and provided written, informed consent. Protocols were approved by the University of Texas, Southwestern Medical Center Internal Review Board on Human Participants Research. Demographic data on participant's age, type of epilepsy, hemisphere, and duration of epilepsy is provided in Table 1.

Electrode localization. Localization of electrodes was performed by presurgical planning followed by stereotactic placement of depth electrodes and postoperative fusion of CT images with the preoperative highresolution MR images (MPRAGE, coronally acquired $1 \mathrm{~mm}$ slices) as conducted in our prior work (Lega et al., 2017; Lin et al., 2017). The cortical loci of electrode contacts were identified using Freesurfer's autosegmentation software (Fischl et al., 1999). All contact loci were confirmed by expert neuroradiology review as a part of standard clinical practice. For all analyses, we focused on the electrodes planted in the hippocampus and PCC (Fig. 1). Sixteen of the 17 participants had their stimulation sites in the left PCC and one participant had their stimula- 
Table 1. Demographic data indicating participant's age, type of epilepsy, hemisphere, and duration of epilepsy across all participants $(N=17)$ in the study

\begin{tabular}{llllc}
\hline Subject no. & Age & Type of epilepsy & Hemisphere & Duration of epilepsy (y) \\
\hline 1 & 66 & Left MTL & LH & 7 \\
2 & 35 & Right NTL & RH & 28 \\
3 & 31 & Right NTL & RH & 3 \\
4 & 42 & Left MTL & LH & 11 \\
5 & 26 & Multifocal & B & 8 \\
6 & 45 & Left MTL & LH & 22 \\
7 & 42 & Bilateral MTL epilepsy & B & 5 \\
8 & 23 & Right NTL & RH & 10 \\
9 & 24 & Left MTL & LH & 5 \\
10 & 42 & Bilateral MTL & B & 9 \\
11 & 21 & Multifocal & B & 4 \\
12 & 39 & Multifocal & B & 15 \\
13 & 52 & Right MTL & RH & 44 \\
14 & 39 & Bilateral NTL & B & 17 \\
15 & 45 & Left NTL & LH & 23 \\
16 & 36 & Left NTL and extratemporal & LH & 26 \\
17 & 35 & Left MTL & LH & 6 \\
\hline
\end{tabular}

NTL, Neocortical temporal lobe; LH/RH, left/right hemisphere; B, bilateral.

tion site in the right PCC. For stimulation, all electrodes were targeted to the retrosplenial region of the PCC, using the splenium of the corpus callosum as a landmark for placement. This incorporated Brodmann areas 26, 29, 30, and the ventral aspect of area 23. Across all participants, the total number of hippocampal contacts was 118 , with an average of 6.94 electrodes per participant and the total number of PCC electrodes was 43 , with an average of 2.86 electrodes per participant. The stimulation protocol applied during the study phase of the episodic memory task included a $25 \mathrm{~s}$ period of high-frequency stimulation. Stimulation was applied with a frequency of $100 \mathrm{~Hz}$ using the Grass S88 stimulator (Grass Technologies). We used bipolar pairs of electrodes for stimulation, the deepest contact of which had been localized to the PCC. Stimulation parameters were determined using accepted safety thresholds for DBS drawn from initial work (Butson and McIntyre, 2007), and by incorporating typical parameters used for DBS techniques (Suthana et al., 2012; Jacobs et al., 2016). The critical safety threshold that is generally accepted is $30 \mu \mathrm{C} / \mathrm{cm}^{2} /$ phase. Here, we used biphasic matched-square wave pulses with a pulse width of $200 \mu \mathrm{s}$. The surface area of depth electrodes is $\sim 0.05$ $\mathrm{cm}^{2}$.

Experimental design and statistical analysis. Our goal was to test the effect of stimulation on episodic memory performance when stimulation was applied during encoding. Similar to prior iEEG studies (Jacobs et al., 2016; Goyal et al., 2018), we used a free recall task in which participants were sequentially presented with a list of study words under the instruction to memorize them for a subsequent recall phase. The recall phase was preceded by a distractor task that required solving arithmetic problems for $20 \mathrm{~s}$. Participants were then given $30 \mathrm{~s}$ to verbally recall words from memory in any order (Fig. 2a). The words used in the word lists were chosen from a pool of high-frequency nouns. The entire list is available at: https://memory.psych.upenn.edu/WordPools. There were a total of 20 lists and each list comprised of 10 words. Each word in the study list was presented for $1500 \mathrm{~ms}$, with an intertrial interval of 1000 ms. Stimulation to the PCC was applied during the entire duration of the encoding phase of $50 \%$ of the study lists (we refer to these lists as stimulation during encoding [ES] lists), whereas no stimulation was applied during encoding phase of the remaining word lists (we refer to these lists as no stimulation [NS] lists). Stimulation to PCC was synchronized with the onset of the first word in each ES list and was delivered throughout the encoding period to largely avoid concerns of poststimulation electrophysiological changes affecting oscillatory patterns of nearby items. To maintain equivalence between stimulation and non-stimulation trials, we used the following steps: (1) Both types of trials were interleaved throughout the experimental session; (2) items from the word pool were randomly assigned to stimulation and non-stimulation word lists with no differences in semantic content; (3) during stimulation, we made sure that all physical connections as well as the appearance of the stimulator gave no indication of whether stimulation was applied; and (4) during the experiment, word lists were scored by a research assistant blinded to the stimulation condition.

To test for behavioral effects of stimulation on episodic memory, for ES and NS study lists, separately, we measured the following: (1) the percentage of correctly recalled words by evaluating the number of correctly recalled words (i.e., only words within a given list without intrusions from prior lists) across all lists in a condition (NS/ES) and dividing by the total number of words across all lists in a condition (NS/ES); (2) the total number of intrusions; (3) the percentage recall of items based on their position (first to 10th separately) in the study list; (4) primacy effect by evaluating percentage recall of primacy item (first item) versus the percentage recall of remaining items; (5) the temporal clustering factor (TCF), which is a measure of participant's tendency to cluster the recalled items based on their temporal proximity during the study period (Polyn et al., 2009a,b; TCF measures the tendency for recall transitions to occur between items presented at nearby list positions: TCF of 1 indicates perfect temporal contiguity, with the participant only making transitions to temporally adjacent items, whereas a TCF of 0.5 indicates that the participant is making random transitions; Polyn et al., 2009a); and (6) the semantic clustering factor (SCF), which is a distance measure of participant's tendency to cluster recall items based on their semantic associations (Polyn et al., 2009a,b; based on a distance matrix provided by Latent Semantic Analysis; Landauer et al., 1997). Both TCF and SCF were calculated using the behavioral toolbox developed at the University of Pennsylvania (http://memory.psych.upenn.edu/Behavioral_toolbox).

All statistical analysis, including $t$ tests, repeated-measures ANOVAs, and Pearson's correlation, were performed using MATLAB. Error bars in Figure $2 c$ represent SEM.

Oscillatory power analysis. Using the stereotactic EEG implantation technology, which allowed simultaneous recordings from hippocampal electrodes while stimulation was delivered to the PCC, our goal was to test whether stimulation of PCC induced modulations in hippocampal oscillations. First, we compared oscillatory power in the hippocampus during the presentation of all items (including successfully encoded items and unsuccessfully encoded items) in the ES versus NS conditions. For this purpose, we conducted a power analysis. Signal from the hippocampal electrodes was sampled at $1 \mathrm{kHz}$ on a Nihon-Kohden 2100 clinical system under a bipolar montage with the most medial white matter contact on individual electrodes as the reference (for hippocampal recordings, this was white matter in the adjacent subcortical temporal lobe). Before the power analysis, we performed the following quality checks. We used an automated artifact rejection algorithm to exclude noise and spiking activity. We excluded activity from electrodes that were at the site of seizure onset locations or frequent interictal activity using kurtosis algorithm (with threshold of 4 ) to exclude abnormal events. We used a bipolar referencing scheme to avoid the possibility of stimulation artifact from affected electrodes modifying signal at other locations. As stimulation was applied at $100 \mathrm{~Hz}$, we used an adaptive notch filter to remove any stimulation-related noise artifacts $>100 \mathrm{~Hz}$. After the quality checks, power analysis was conducted using methods used in our prior work (Lega et al., 2012). Power values were extracted from $1600 \mathrm{~ms}$ time windows following the appearance of the study item using Morlet wavelets with a width of six at $49 \mathrm{log}$-spaced frequencies between 2 and $128 \mathrm{~Hz}$. Once power was extracted, we compared the frequency-time windows in two stages: First, per participant, we performed a statistical comparison between oscillatory power elicited by study items in the ES lists versus those in the NS lists by generating $t$-statistics at each frequency-time pixel per electrode contact. Performing statistical tests within electrodes allowed us to then average data across electrodes using the statistic and avoided the need to normalize power values. Second, across all participants, the distribution of $t$-statistics was then compared against the null hypothesis using a statistical $t$ test, resulting in $p$-values for each time window and frequency. We then converted the $p$-values to $z$-scores using normal inverse cumulative distribution and significant frequency band clusters across participants were obtained at $p<0.05$ or $z>1.65$ corrected for multiple comparisons.

SME analysis. This analysis was conducted to determine how oscillatory power differed during the encoding of later successfully recalled versus forgotten items (Paller and Wagner, 2002) as a function of the ES 
versus NS manipulation. For each participant, we indexed SMEs with $t$-statistics by contrasting oscillatory power (at each electrode, frequency, and time window) elicited by successfully recalled items versus forgotten items, and obtained average $t$-statistics across all hippocampal electrodes. We calculated a "delta SME" as SME in NS minus SME in ES conditions and then tested the distribution of these values against the null hypothesis of a zero difference using statistical $t$ test. Resulting $p$-values for each time window and frequency were converted to $z$-scores using the normal inverse cumulative distribution and significant frequency band clusters across participants were obtained at $p<0.05$ or $z>1.65$ corrected for multiple comparisons.

Analysis of relationship between behavioral measures and hippocampal oscillations. One of the goals of our study was to test whether there is a relationship between stimulationinduced changes in memory performance and stimulation-induced modulations in hippocampal oscillations. To evaluate this, per participant, we first averaged the $t$-statistics (obtained from the prior two analyses: i.e., NS vs ES comparison, and delta SME measures) across the following bands: theta $(2-10 \mathrm{~Hz})$, alpha $(10-14 \mathrm{~Hz})$, beta $(15-24$ $\mathrm{Hz})$, low gamma $(25-40 \mathrm{~Hz})$, and high gamma $(>40 \mathrm{~Hz})$ bands. Thus, per participant, we obtained two hippocampal oscillatory measures (per frequency band). Next, we correlated these measures with the participants' behavioral memory performance to obtain a relationship between behavior and hippocampal activations.

Analysis of baseline functional connectivity between hippocampus and PCC electrodes. One of the factors that can drive stimulation effects is the functional connectivity between the stimulated and another brain region (Matsumoto et al., 2004; Foster et al., 2013, 2015; Gu et al., 2015; Solomon et al., 2018). The PCC is anatomically connected to the hippocampus via the cingulum bundle, and prior research suggests that abnormal functional connectivity between these two regions may lead to cognitive impairments (Zhou et al., 2008). We investigated whether baseline functional connectivity (that is functional connectivity measured when no stimulation is delivered) between PCC and hippocampus predicts the effects of stimulation on recall memory performance and oscillatory activity in the hippocampus. Although in our free recall experiment no stimulation was delivered during the NS condition, we were unable to use these trials to test for baseline PCC-hippocampal connectivity because throughout the stimulation session, PCC electrodes were connected to the stimulation device exclusively. This permitted artifact free recording from adjacent regions, namely hippocampus, without concerns for amplifier saturation, but precluded performing connectivity analyses during NS trials. Thus, we conducted a follow-up free recall experiment in the absence of stimulation. This provided a baseline condition to examine neural connectivity between the hippocampus and PCC. A subset of our participants (11 of 17) participated in this follow-up experiment.

To measure functional connectivity between the hippocampal and PCC electrodes for each participant $(n=11)$, we obtained phase-locking values (PLVs) (Lachaux et al., 1999; Foster et al., 2013; Lega et al., 2017; Lin et al., 2017) for each frequency band: theta $(2-10 \mathrm{~Hz})$, alpha (10-14 $\mathrm{Hz})$, beta $(15-24 \mathrm{~Hz})$, low gamma $(25-40 \mathrm{~Hz})$, and high gamma $(>40$ $\mathrm{Hz}$ ). PLV is a commonly used method to estimate functional connectivity and it is operationalized as phase synchronization between two brain regions. Phase-locking analysis is well suited for connectivity measures as it provides an estimate of temporal relationships between neural signals, independent of their amplitude (Lachaux et al., 1999). Specifically, PLV estimates the across-trial consistency of phase difference between two time series for each frequency and condition. Calculation of phase synchrony was implemented with Rayleigh tests including a shuffle procedure (200 shuffles) for randomization, generating a phase locking statistic as described previously (Lachaux et al., 1999). A PLV from distribution of phase differences for successfully recalled and nonrecalled/ forgotten words was obtained for each electrodes pair. Calculation of oscillatory synchrony was performed with a Rayleigh test applied to the distribution of phase differences at each frequency-time pixel across all items separately for successfully recalled and forgotten items (Palva et al., 2005). Specifically, a subsequent memory effect in phase synchrony was analyzed by running a $t$ test between distributions of $z$-values of recalled events and nonrecalled events for all contact pairs from all subjects. This resulted in a $p$-value, which was converted to a $z$-score at every frequency-time step. To estimate chance level, we randomly selected a subset of subsequently nonrecalled items to balance the number of successful and unsuccessful encoding trials for estimating phase synchrony. Per electrode pair, we extracted functional connectivity measures (comparison between recalled and nonrecalled distributions of phase synchrony values) as $z$-scores, across frequency-time bands, and then averaged all the connectivity measures across electrode pairs within each band. Finally, to test whether functional connectivity between PCC and 
hippocampus would predict the effects of stimulation on recall performance memory and oscillatory activity in the hippocampus, we correlated participant's stimulation-induced behavioral and oscillatory measures with their functional connectivity measure.

\section{Results}

\section{Stimulation of PCC impairs verbal recall memory}

To determine whether stimulating the PCC modulates memory encoding, we used a free recall memory paradigm in which PCC was stimulated during half of the study lists (Fig. 2a). First, consistent with prior studies (Foster and Parvizi, 2017), stimulation applied across the entire study list did not elicit any reports of subjective experiences, and nor was there evidence of induced seizures or other adverse consequences. We then compared recall performance, measured as the percentage of correctly recalled study words, during the ES versus NS conditions. Results revealed that PCC stimulation during encoding significantly impaired recall performance (mean \pm SD: $\mathrm{M}_{\mathrm{NS}}=28.9 \pm 10.24 \%$, $\mathrm{M}_{\mathrm{ES}}=25.3 \pm 10.26 \%, t_{(16)}=2.62, p<0.01$, two-tailed, Fig. $2 b$ ). The direction of change (i.e., memory disruption following PCC stimulation) is consistent in $70.5 \%$ of our subjects (Fig. $2 b$, solid lines). In terms of the number of items, for the $70.5 \%$ participants whose memory was impaired during stimulation, they typically remembered an average of six items fewer than they did when stimulation was not present (of a total of 100 words across 10 lists in each condition). We also found that stimulation was not accompanied by an increased intrusion of recall words from prior lists, as this was comparable across NS and ES conditions $\left(\right.$ mean $\pm \mathrm{SD}: \mathrm{M}_{\mathrm{NS}}=4.17 \pm 3.39, \mathrm{M}_{\mathrm{ES}}=4.52 \pm 3.62, t_{(16)}=0.49$, $p=$ ns).

Next, we investigated whether the stimulation-related recall impairment demonstrated a serial order effect. Specifically, we examined performance separately for primacy items (items in first position in each 10-word list) and non-primacy items (items in second to $10^{\text {th }}$ positions in a list). Serial position curves are shown in Figure $2 c$. First, consistent with longstanding findings (Murdock, 1962), recall probability for primacy items was higher than that for non-primacy items (main effect of item position in a 2-way repeated-measures ANOVA with factors item position (primacy/non-primacy items) and stimulation condition (ES/ NS), $\left.F_{(1,336)}=49.24, p<0.001\right)$. Importantly, PCC stimulation affected recall of primacy items to a greater extent than recall of non-primacy items (a significant interaction between item position and stimulation condition: $\left.F_{(1,336)}=4.08, p<0.05\right)$. Specifically, recall memory for early items in ES lists was lower than that in NS lists $\left(t_{(16)}=3.98, p<0.001\right)$, but was largely comparable for late items $\left(t_{(16)}=1.46, p=n . s.\right)$.

In a free recall task, participants tend to remember items in the order that they were presented at study (Howard and Kahana, 2002). Participants also have a tendency to cluster items based on their semantic association. Thus, we investigated whether stimulating the PCC disrupted the temporal or semantic organization of recall. We estimated the TCF (see Materials and Methods for description of quantification), which is a measure of a participant's tendency to cluster recalled items based on their temporal proximity during study. In contrast to recall performance, which decreased when PCC was stimulated, TCF was greater in ES than NS conditions $\left(t_{(16)}=2.13, p=0.04\right.$, Fig. $\left.2 d\right)$. Given the fact that stimulation affected recall of primacy items, next we investigated whether perhaps it was also the case that temporal clustering increased when analyzed only for blocks when primacy recall was successful. However, we found no difference in TCF when analyzed only for lists in which primacy encoding succeeded, indi- cating that perhaps lists on which primacy items were disrupted were those that exhibited a TCF increase. We also measured the SCF (see Materials and Methods), a distance measure of participants' tendency to cluster recall items by semantic associations. Results showed comparable SCF in ES and NS lists $\left(t_{(16)}=0.18\right.$, $p=$ ns, Fig. $2 e$ ).

Combined, our results showed that PCC stimulation impaired episodic recall memory and in particular, memory for early items in the study lists. However, the temporal and semantic clustering factors related to recall memory either showed an opposite effect during stimulation (TCF) or remained largely unaffected (SCF) by stimulation.

\section{Stimulation of PCC increases gamma-band and decreases theta-band power in the hippocampus across all serial position items}

Next using the unique opportunity afforded by stereotactic EEG implantation to record oscillations from the hippocampus while stimulating the PCC, we tested whether PCC stimulation affected hippocampal activity. Results revealed that stimulation of the PCC modified hippocampal activity (Fig. $3 a$ shows the direction of power changes in a sample participant across low and highfrequency bands). Specifically, we found that hippocampal power was significantly lower in the theta band $(2-10 \mathrm{~Hz})$ but higher in the low $(25-40 \mathrm{~Hz})$ and high gamma bands $(>40 \mathrm{~Hz})$ in the ES compared with NS condition (significant at a $p<0.05$ or $z=1.64$ level corrected for multiple comparisons). Given our behavioral findings that recall was impaired predominantly for primacy items, we also examined hippocampal oscillatory power for primacy and non-primacy items in the ES versus NS conditions. Regardless of the serial position, stimulation induced increases in power in low and high gamma bands, but decrease in power in theta band. This result indicates that stimulation likely induces uniform oscillatory changes in the hippocampus across all study lists, despite a behavioral effect that was confined to early occurring items.

Next, we investigated how hippocampal oscillatory SMEs (successfully recalled vs forgotten items) differed between the ES and NS conditions. For this, we examined the power difference associated with encoding success rather than across all trials (in contrast to the analysis described above, which did not distinguish effects according to encoding success). We obtained the following results: First, we observed an increase in low gamma, high gamma, and beta band power and a decrease in theta band power in both the NS and ES conditions for successfully remembered items versus forgotten items (positive and negative SMEs, respectively) (Fig. 3b). This result is consistent with our expectations for hippocampal and temporal oscillations based on prior intracranial research (Sederberg et al., 2003; Lega et al., 2012; Long et al., 2014). However, our data showed that stimulation modified the hippocampal subsequent memory effect, with a significant reduction in the low, high, and beta band SMEs, but an enhancement of the theta band SME (significant at a $p<0.05$ or $z=1.64$ level corrected for multiple comparisons). Combined, our results revealed that stimulation to PCC modulated hippocampal activity, by inducing an overall increase in gamma and decrease in theta band power across all items. However, stimulation also induced a reduction in the magnitude of the relative gamma power increase and an increment in the magnitude of relative theta power decrease, that typically occur during successful memory encoding (Long et al., 2014). 


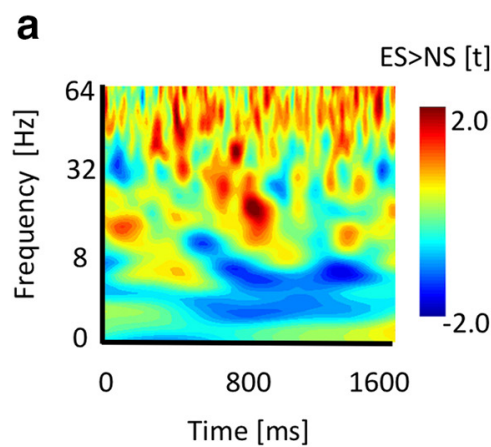

C

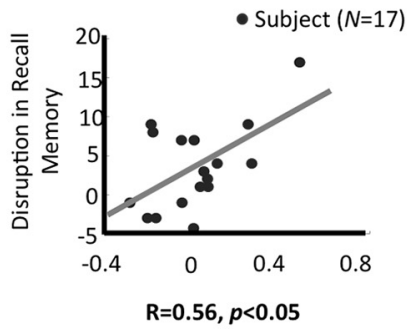

Stimulation-induced hippocampal modulations in low gamma-band $[\mathrm{t}]$

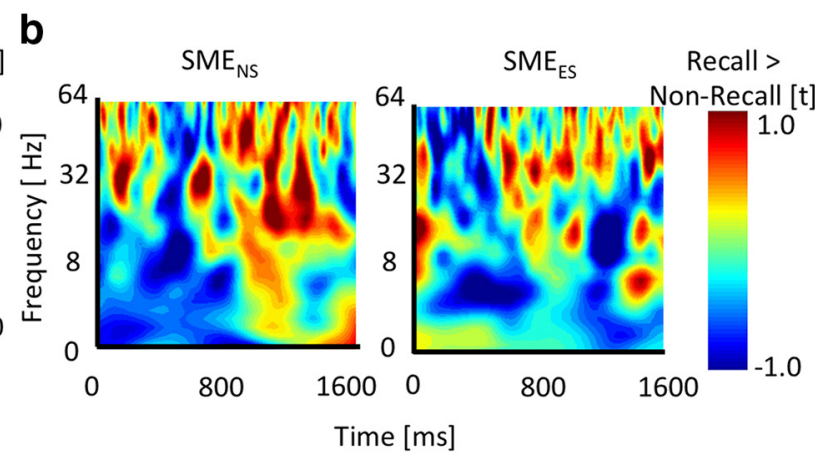

d

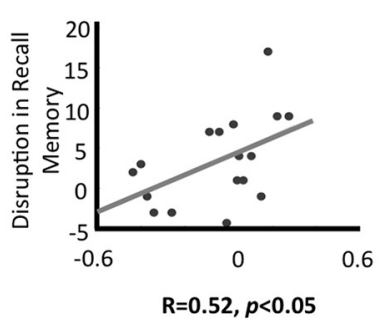

e

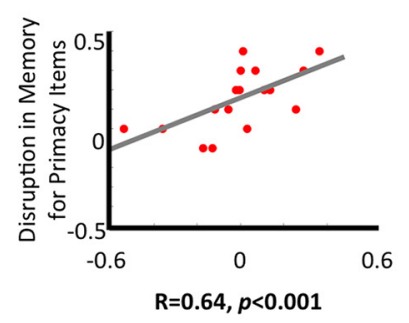

Delta SME in low gamma band $\left(\mathrm{SME}_{\mathrm{NS}}-\mathrm{SME}_{\mathrm{ES}}\right)$

Figure 3. Stimulation induces changes in low gamma and theta band oscillations in the hippocampus and neural changes are linked to behavioral memory disruptions. $\boldsymbol{a}$, Frequency-time plot of a sample participant showing increases in low and high gamma band power and decrease in theta band power during ES versus NS conditions. $\boldsymbol{b}$, Frequency-time plot of a sample participant showing power differences when an item is successfully recalled versus forgotten (successful memory effect, SME) in ES (left) and NS (right) conditions. Gamma power increased whereas theta power decreased in both cases. For $\boldsymbol{a}, \boldsymbol{b}: \mathrm{Y}$-axis represents frequency $[\mathrm{Hz}] ; \mathrm{X}$-axis represents time [ms]. c, Significant positive correlation between stimulation induced hippocampal gamma power changes (measured as $t$-statistics of the contrast between ES versus NS conditions) and disruption in the memory (measured as percentage recall during NS minus ES conditions). In $\boldsymbol{d}$ and $\boldsymbol{e}$, there was a significant positive correlation between delta hippocampal gamma SME (measured as follows: SME in NS minus SME in ES condition) and disruption in memory performance (measured as follows: percentage recall of all items/percentage recall of primacy items in NS minus that in ES condition).

\section{Are neural modulations in gamma and theta activity related to memory performance?}

The results described thus far show that PCC stimulation disrupted behavioral memory performance and modulated hippocampal activity. Next, we investigated whether there was a relationship between stimulation-induced changes in memory performance and hippocampal power across participants. To address this question, we correlated measures of hippocampal neural modulation with the magnitude of stimulation-induced changes in recall performance. We measured the latter as the difference in recall performance between NS versus ES conditions for all study items, and separately analyzed the effect on primacy items. We operationalized stimulation-induced neural changes as average contrasts (between NS and ES conditions) of power estimates in different frequency bands during all trials (successful and unsuccessful), and the subsequent memory effect in these frequency bands (successful vs unsuccessful power differences) (see the Materials and Methods). Results revealed that there was a significant positive relationship between behavioral and neural effects of stimulation in the low gamma bands $(R=$ $0.56, p<0.05$, Fig. $3 c$ ). The $95 \%$ confidence interval measured using bootstrapping estimate for the correlation coefficient between behavioral and neural effects of stimulation is [0.1 0.88 ]. Greater positive values correspond to a greater reduction of memory performance due to stimulation. Specifically, participants with greater stimulation-induced reduction in recall memory showed a larger increase in low gamma power. A similar relationship between behavioral and neural effects of stimulation was not evident for theta or high gamma bands or when we tested this relationship for just the primacy items $(R<0.32, p=n s)$, consistent with our previous observation that hippocampal oscillatory changes persist across the stimulation period.

Using the subsequent memory contrast, we also found a significant positive relationship between behavioral impact of stimulation and oscillatory difference in the low gamma band $(R=$ $0.52, p<0.05,95 \%$ confidence interval using bootstrap estimate: [0.2 0.76], Fig. $3 d$ ). A similar significant positive relationship was found between disruption in recall memory for primacy items (excluding other serial positions from the behavioral measurement) and stimulation-induced SME changes in low gamma $(R=0.64, p<0.001,95 \%$ confidence interval: [0.43 0.8], Fig. $3 e)$ and beta $(R=0.70, p<0.001,95 \%$ confidence interval: $[0.5$ $0.85]$ ) bands. No significant relationship between neural and behavioral effects of stimulation was evident in other frequency band SMEs ( $R s<0.04, p s=\mathrm{ns})$.

As a control, to test whether stimulating PCC also affected the oscillatory activity in the hippocampus during the distractor arithmetic task, in the NS versus ES condition, we repeated the oscillatory analysis by evaluating the hippocampal responses during the distractor task and correlating neural with behavioral recall memory performance. We found no significant correlation between the hippocampal activation during the math task and recall memory performance across the frequency bands (theta $(2-10 \mathrm{~Hz})$, alpha $(10-14 \mathrm{~Hz})$, beta $(15-24 \mathrm{~Hz})$, low gamma $(25-40 \mathrm{~Hz})$, and high gamma $(>40 \mathrm{~Hz})$ bands, $R s<0.29$, $p s=\mathrm{ns})$.

Combined, our results suggest that PCC stimulation during word encoding results in specific changes in the hippocampal neural activity that were consistent across subjects, and that stimulation-induced hippocampal modulations across all trials, 
a
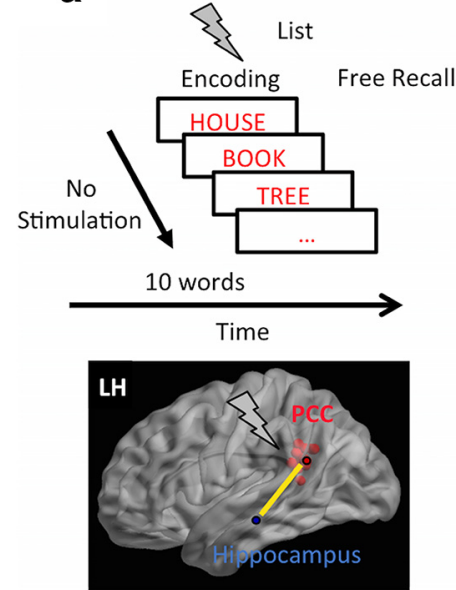

b

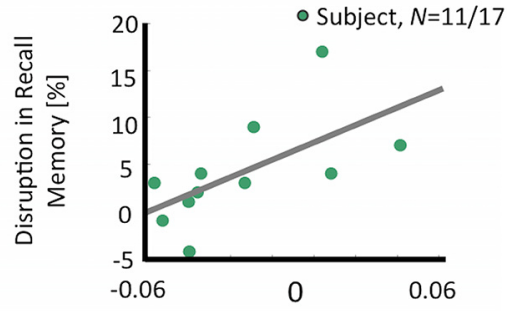

Hippocampal-PCC functional connectivity

$\mathrm{R}=0.62, p<0.05$

Figure 4. Baseline PCC-hippocampal functional connectivity is positively linked to stimulation-induced memory disruptions. $\boldsymbol{a}, \mathrm{PCC}-$ Hippocampal functional connectivity, measured as oscillatory synchrony between hippocampal electrodes and PCC electrodes (in a subset of participants, $n=11$ of 17 ) in a second free recall experiment in the absence of stimulation. $\boldsymbol{b}$, Positive correlation between baseline functional connectivity and stimulation-induced recall memory disruption. That is, participants with higher PCC-hippocampal functional connectivity tended to show a larger disruption in memory performance due to stimulation.

predominantly in the low gamma band, predict behavioral memory disruptions.

\section{Does connectivity between hippocampus and PCC predict the behavioral effects of stimulation?}

A factor that can drive stimulation effects in the brain is interregional functional connectivity. Therefore, we investigated whether functional connectivity between the PCC and hippocampus predicted stimulation effects on recall memory and hippocampal activity. We sought to measure baseline functional connectivity when no stimulation was present. In a subset of our participants ( $n=11$ of 17 participants), connectivity values were obtained in a separate, non-stimulation experimental session while participants performed a free recall task (Fig. 4a). Per participant, we first obtained connectivity measures between the hippocampal and PCC electrodes in the theta, alpha, beta, low, and high gamma bands (see Materials and Methods) and then we correlated connectivity values with stimulation-induced changes in recall performance for all trials, low gamma oscillatory power changes across all trials (as in Fig. $3 a$ ), and subsequent memory effects (as in Fig. $3 b$ ). We found that only baseline functional connectivity in the low gamma band was related to stimulationinduced changes in memory responses. Specifically, participants with larger PCC-hippocampal functional connectivity in the low gamma band showed greater reduction in episodic recall memory during stimulation $(R=0.62, p<0.05,95 \%$ confidence interval using bootstrapping: [0.43 0.90]; Fig. $4 b$ ). There were also trends for positive relationships between low gamma-band connectivity and stimulation-induced low gamma power differences $(R=$ $0.42, p=0.1)$ and SME-related low gamma power changes $(R=$ $0.52, p=0.08$ ). However, connectivity in other frequency bands did not predict the behavioral or oscillatory impact of stimulation ( $p=\mathrm{ns}$ ). Overall, our results demonstrated that functional connectivity between hippocampus and PCC electrodes might be linked to the behavioral effects of stimulation.

\section{Discussion}

Using DBS, our study examined the role of the PCC in episodic memory encoding. Our results revealed that stimulating the PCC during memory encoding impairs subsequent memory performance and results in modulations in hippocampal oscillatory power. These modulations manifest as increases in low gamma and decreases in theta power. These effects on hippocampal activity were also accompanied by stimulation-induced reduction in the hippocampal low gamma SME. Importantly, these neural modulations in the hippocampus were linked to observed reductions in memory performance and functional connectivity between the PCC and hippocampus predicted stimulationrelated effects on recall performance. Combined, our results provide causal evidence for the importance of PCC in episodic memory encoding and indicate that memory can be modulated selectively via stimulation of brain structures in the extended hippocampal memory network.

Our key finding is that high-frequency stimulation delivered during encoding resulted in a decrease in recall performance relative to the no stimulation condition. Memory disruption was predominantly found for early compared to late items in the study lists. These findings of memory disruption following PCC stimulation were surprising given that fMRI studies show negative SMEs for successful versus unsuccessful encoding in the posterior cingulate (Otten and Rugg, 2001; Daselaar et al., 2004; Wagner et al., 2005; Miller et al., 2008; Duverne et al., 2009; Huijbers et al., 2011). In fact, these prior results led to our prediction that stimulating PCC during encoding might enhance (Suthana et al., 2012; Suthana and Fried, 2014; Kucewicz et al., 2018) but not disrupt memory. Rather, our behavioral results are reminiscent of prior reports of memory impairment during stimulation of the hippocampus and entorhinal cortex (Coleshill et al., 2004; Lacruz et al., 2010; Jacobs et al., 2016; Goyal et al., 2018). Consistent with our findings, stimulating regions in the MTL also disrupted memory for early more so than later items in a list (Goyal et al., 2018). However, in contrast, stimulating the MTL negatively affected both the temporal and semantic clustering factors (Goyal et al., 2018), which are contradictory to our findings of the small enhancement in TCF and no change in SCF during PCC stimulation. Combined, our findings provide evidence for a causal role for the PCC during memory encoding, and, we infer that the PCC plays a specific and more limited role than the MTL.

What is the role of the PCC in memory encoding? The novelty of our study lies in the fact that using the stereo-EEG technique allowed us to test the effect of direct stimulation to deep brain regions such as the PCC on free recall memory and simultaneously evaluate effects of indirect stimulation on hippocampal electrodes. Stimulation to PCC not only affected recall memory, but it also modulated hippocampal oscillations. This leads to two questions: (1) to what degree are our observations attributable to contributions of the PCC itself to episodic encoding? and (2) to what degree are the results attributable to PCC's downstream impact on the hippocampus? In the absence of permanent lesions, disentangling these issues is challenging, nonetheless, we speculate that the impact of PCC stimulation may represent a mixture of these factors. This is because, whereas hippocampal oscillatory changes were correlated with effects on recall, specific 
behavioral effects were not entirely predicted by hippocampal changes. Thus, the PCC may be a component in a larger memory circuit, and behavioral alteration will result in manipulation of the circuit as a whole.

Our behavioral results may be consistent with a model by which the PCC supports elaborative encoding representations elicited by item rehearsal. We observed a greater impact on the encoding of primacy items than later items. Prior work shows that the primacy effect arises due to rehearsal for early items in the study list during the presentation of subsequent items (Brodie and Murdock, 1977) and that rehearsal provides an encoding boost for these items (Sederberg et al., 2006). One theory of PCC functioning posits that the PCC may be necessary for the integration of episodic information with existing semantic knowledge, forming a useful (and possibly well consolidated) representation of an item (a schema) (Bird et al., 2015), thereby reducing the episodic characteristics of such memories. Insofar as the process of rehearsal may link memory items to semantic representations (possibly forming a schema), our data are consistent with this idea, as stimulation preferentially disrupted primacy encoding. Further, a key observation that distinguishes PCC stimulation from MTL stimulation is that temporal clustering was not disrupted as in prior studies of hippocampus (Goyal et al., 2018). In fact, TCF was mildly enhanced with PCC stimulation and SCF was unaffected, which also suggests an independent contribution of PCC toward episodic memory processing, separate from hippocampus. Thus, if our observations of memory disruption were exclusively attributable to downstream hippocampal effects, then the pattern of behavioral changes would match those observed with hippocampal stimulation. Related to this point, consistent with our findings of specific disruption for encoding with PCC stimulation, PCC's contributions to encoding are not visible in univariate analyses of BOLD signal changes but are nonetheless significant.

Our data also provide evidence that the hippocampus and PCC interact during memory encoding (Maddock, 1999; Ranganath et al., 2005; Zhou et al., 2008) and it is unlikely that the PCC could fulfill a putative role in supporting representations of rehearsed items without hippocampal contribution. In fact, our findings that baseline PCC-hippocampal connectivity is related to the impact of stimulation on memory encoding and that memory disruption is correlated with the magnitude of hippocampal gamma power changes provide support for this interpretation. Interestingly, stimulation of PCC elicited a gamma power increase and a theta power decrease across all items, but the oscillatory patterns characteristic of successful encoding (positive gamma SME; Long et al., 2014) were disrupted by PCC stimulation. Thus, the impact of PCC stimulation on the hippocampal gamma SME indicates that PCC may contribute toward generation of these oscillatory responses and that stimulation to PCC, when delivered to an optimally functioning memory system, may disrupt more subtle high-frequency gamma activity associated with memory tasks (Kahana, 2006; Lachaux et al., 2012; Kucewicz et al., 2014). Prior iEEG studies of posterior medial cortex, including the PCC and retrosplenial cortex (Foster et al., 2012, 2013; Foster and Parvizi, 2017; Daitch and Parvizi, 2018) provided an important bird's-eye view of high gamma activation related to memory cognition, which we replicate, however, our study further extends the PCC's role in memory encoding and item rehearsal and its potential impact on hippocampal gamma activity. One limitation of our study is that we are not able to assess differential hemispheric effects of stimulation as a majority of participants had their stimulation sites in the left hemisphere.
Future work can test for hemispheric effects of stimulation on behavior and neural activity.

A long-term goal of iEEG research is to explore novel brain targets for neuromodulation and to develop strategies that allow brain-machine interface devices to improve cognitive performance in patients suffering from cognitive decline. Although we did not observe memory enhancement with stimulation, our results highlight that the PCC, in combination with the hippocampus and entorhinal cortex, may be a propitious network for open- and closed-loop (Rosin et al., 2011; Ezzyat et al., 2018) responsive stimulation strategies. Our data are the first to suggest that extended stimulation epochs can be used to safely modulate hippocampal activity with significant implications on recall performance and that stimulation outside of the MTL can be systematically applied to study networks of episodic memory processing. More generally, our study describes a method using stereo-EEG electrode arrays to elucidate the lesser-known contributions of deep cortical regions for cognitive and memory processing and can serve as a model for researchers testing alternative strategies for neuromodulation of memory. Although our connectivity results are based on a subset of participants, they highlight the predictive power of functional connectivity and its utility in understanding how stimulating a network hub might affect neural responses (Solomon et al., 2018) and behavioral performance. Functional connectivity can be used as a critical measure for determining whether an individual may be a candidate for DBS related therapeutic strategies. Future work combining task-specific and/or resting-state neuroimaging measures with electrophysiological measures (Nir et al., 2008; Keller et al., 2013; Foster et al., 2015; Kucyi et al., 2018) may provide a better understanding of functional connectivity between deep brain regions and this a priori information can be beneficial for developing closed-loop stimulation strategies.

In conclusion, we present novel human data in which we explored the mnemonic functions of the PCC using deep brain stimulation. We (1) demonstrate the feasibility of applying extended periods of electrical stimulation to a deep cortical region such as the PCC to modulate memory, (2) explicate potential causal role for the PCC during episodic memory encoding, and (3) highlight functional connectivity as an informative measure of how stimulation might affect memory performance, with implications for therapeutic advancements in memory diseases.

\section{References}

Bird CM, Keidel JL, Ing LP, Horner AJ, Burgess N (2015) Consolidation of complex events via reinstatement in posterior cingulate cortex. J Neurosci 35:14426-14434.

Brodie DA, Murdock BB (1977) Effects of presentation time on nominal and functional serial position curves in free recall. Journal of Verbal Learning and Verbal Behavior 16:185-200.

Bronstein JM, Tagliati M, Alterman RL, Lozano AM, Volkmann J, Stefani A, Horak FB, Okun MS, Foote KD, Krack P, Pahwa R, Henderson JM, Hariz MI, Bakay RA, Rezai A, Marks WJ Jr, Moro E, Vitek JL, Weaver FM, Gross RE, et al. (2011) Deep brain stimulation for parkinson disease: an expert consensus and review of key issues. Arch Neurol 68:165.

Butson CR, McIntyre CC (2007) Differences among implanted pulse generator waveforms cause variations in the neural response to deep brain stimulation. Clin Neurophysiol 118:1889-1894.

Cabeza R, Daselaar SM, Dolcos F, Prince SE, Budde M, Nyberg L (2004) Task-independent and task-specific age effects on brain activity during working memory, visual attention and episodic retrieval. Cereb Cortex 14:364-375.

Coleshill SG, Binnie CD, Morris RG, Alarcón G, van Emde Boas W, Velis DN, Simmons A, Polkey CE, van Veelen CW, van Rijen PC (2004) Materialspecific recognition memory deficits elicited by unilateral hippocampal electrical stimulation. J Neurosci 24:1612-1616. 
Daitch AL, Parvizi J (2018) Spatial and temporal heterogeneity of neural responses in human posteromedial cortex. Proc Natl Acad Sci U S A 115:4785-4790.

Daselaar SM, Prince SE, Cabeza R (2004) When less means more: deactivations during encoding that predict subsequent memory. Neuroimage 23:921-927.

De Vogelaere F, Santens P, Achten E, Boon P, Vingerhoets G (2012) Altered default-mode network activation in mild cognitive impairment compared with healthy aging. Neuroradiology 54:1195-1206.

Duverne S, Motamedinia S, Rugg MD (2009) The relationship between aging, performance, and the neural correlates of successful memory encoding. Cereb Cortex 19:733-744.

Ezzyat Y, Kragel JE, Burke JF, Levy DF, Lyalenko A, Wanda P, O’Sullivan L, Hurley KB, Busygin S, Pedisich I, Sperling MR, Worrell GA, Kucewicz MT, Davis KA, Lucas TH, Inman CS, Lega BC, Jobst BC, Sheth SA, Zaghloul K, et al. (2017) Direct brain stimulation modulates encoding states and memory performance in humans. Curr Biol 27:1251-1258.

Ezzyat Y, Wanda PA, Levy DF, Kadel A, Aka A, Pedisich I, Sperling MR, Sharan AD, Lega BC, Burks A, Gross RE, Inman CS, Jobst BC, Gorenstein MA, Davis KA, Worrell GA, Kucewicz MT, Stein JM, Gorniak R, Das SR et al. (2018) Closed-loop stimulation of temporal cortex rescues functional networks and improves memory. Nat Commun 9:365.

Fell J, Staresina BP, Do Lam AT, Widman G, Helmstaedter C, Elger CE, Axmacher N (2013) Memory modulation by weak synchronous deep brain stimulation: a pilot study. Brain Stimul 6:270-273.

Fischl B, Sereno MI, Dale AM (1999) Cortical surface-based analysis II: inflation, flattening, and a surface-based coordinate system. Neuroimage 9:195-207.

Foster BL, Parvizi J (2017) Direct cortical stimulation of human posteromedial cortex. Neurology 88:685-691.

Foster BL, Dastjerdi M, Parvizi J (2012) Neural populations in human posteromedial cortex display opposing responses during memory and numerical processing. Proc Natl Acad Sci U S A 109:15514-15519.

Foster BL, Kaveh A, Dastjerdi M, Miller KJ, Parvizi J (2013) Human retrosplenial cortex displays transient theta phase locking with medial temporal cortex prior to activation during autobiographical retrieval. J Neurosci 33:10439-10446.

Foster BL, Rangarajan V, Shirer WR, Parvizi J (2015) Intrinsic and taskdependent coupling of neuronal population activity in human parietal cortex. Neuron 86:578-590.

Fox KCR, Foster BL, Kucyi A, Daitch AL, Parvizi J (2018) Intracranial electrophysiology of the human default network. Trends Cogn Sci 22:307324.

Goyal A, Miller J, Watrous AJ, Lee SA, Coffey T, Sperling MR, Sharan A, Worrell G, Berry B, Lega B, Jobst BC, Davis KA, Inman C, Sheth SA, Wanda PA, Ezzyat Y, Das SR, Stein J, Gorniak R, Jacobs J (2018) Electrical stimulation in hippocampus and entorhinal cortex impairs spatial and temporal memory. J Neurosci 38:4471-4481.

Greicius MD, Srivastava G, Reiss AL, Menon V (2004) Default-mode network activity distinguishes Alzheimer's disease from healthy aging: evidence from functional MRI. Proc Natl Acad Sci U S A 101:4637-4642.

Gu S, Pasqualetti F, Cieslak M, Telesford QK, Yu AB, Kahn AE, Medaglia JD, Vettel JM, Miller MB, Grafton ST, Bassett DS (2015) Controllability of structural brain networks. Nat Commun 6:8414

Howard MW, Kahana MJ (2002) A distributed representation of temporal context. Journal of Mathematical Psychology 46:269-299.

Huijbers W, Pennartz CM, Cabeza R, Daselaar SM (2011) The hippocampus is coupled with the default network during memory retrieval but not during memory encoding. PLoS One 6:e17463.

Inman CS, Manns JR, Bijanki KR, Bass DI, Hamann S, Drane DL, Fasano RE, Kovach CK, Gross RE, Willie JT (2018) Direct electrical stimulation of the amygdala enhances declarative memory in humans. Proc Natl Acad Sci U S A 115:98-103.

Jacobs J, Miller J, Lee SA, Coffey T, Watrous AJ, Sperling MR, Sharan A, Worrell G, Berry B, Lega B, Jobst BC, Davis K, Gross RE, Sheth SA, Ezzyat Y, Das SR, Stein J, Gorniak R, Kahana MJ, Rizzuto DS (2016) Direct electrical stimulation of the human entorhinal region and hippocampus impairs memory. Neuron 92:983-990.

Johnson MD, Lim HH, Netoff TI, Connolly AT, Johnson N, Roy A, Holt A, Lim KO, Carey JR, Vitek JL, He B (2013) Neuromodulation for brain disorders: challenges and opportunities. IEEE Trans Biomed Eng 60:610624 .
Kahana MJ (2006) The cognitive correlates of human brain oscillations. J Neurosci; 26:1669-1672.

Keller CJ, Bickel S, Honey CJ, Groppe DM, Entz L, Craddock RC, Lado FA, Kelly C, Milham M, Mehta AD (2013) Neurophysiological investigation of spontaneous correlated and anticorrelated fluctuations of the BOLD signal. J Neurosci 33:6333-6342.

Kringelbach ML, Jenkinson N, Owen SL, Aziz TZ (2007) Translational principles of deep brain stimulation. Nat Rev Neurosci 8:623-635.

Kucewicz MT, Berry BM, Miller LR, Khadjevand F, Ezzyat Y, Stein JM, Kremen V, Brinkmann BH, Wanda P, Sperling MR, Gorniak R, Davis KA, Jobst BC, Gross RE, Lega B, Van Gompel J, Stead SM, Rizzuto DS, Kahana MJ, Worrell GA (2018) Evidence for verbal memory enhancement with electrical brain stimulation in the lateral temporal cortex. Brain 141:971-978.

Kucewicz MT, Cimbalnik J, Matsumoto JY, Brinkmann BH, Bower MR, Vasoli V, Sulc V, Meyer F, Marsh WR, Stead SM, Worrell GA (2014) High frequency oscillations are associated with cognitive processing in human recognition memory. Brain 137:2231-2244.

Kucyi A, Schrouff J, Bickel S Foster, BL James M. Shine JM, Josef Parvizi J (2018) Intracranial electrophysiology reveals reproducible intrinsic functional connectivity within human brain networks. J Neurosci 38: $4230-4242$

Lachaux JP, Rodriguez E, Martinerie J, Varela FJ (1999) Measuring phase synchrony in brain signals. Hum Brain Mapp 8:194-208.

Lachaux JP, Axmacher N, Mormann F, Halgren E, Crone NE (2012) Highfrequency neural activity and human cognition: past, present and possible future of intracranial EEG research. Prog Neurobiol 98:279-301.

Lacruz ME, Valentín A, Seoane JJ, Morris RG, Selway RP, Alarcón G (2010) Single pulse electrical stimulation of the hippocampus is sufficient to impair human episodic memory. Neuroscience 170:623-632.

Landauer TK Dumais ST (1997) Solution to Plato's problem: the latent semantic analysis theory of acquisition, induction, and representation of knowledge. Psychol Rev 104:211-240.

Leech R, Sharp DJ (2014) The role of the posterior cingulate cortex in cognition and disease. Brain 137:12-32.

Lega BC, Jacobs J, Kahana M (2012) Human hippocampal theta oscillations and the formation of episodic memories. Hippocampus 22:748-761.

Lega B, Germi J, Rugg M (2017) Modulation of oscillatory power and connectivity in the human posterior cingulate cortex supports the encoding and retrieval of episodic memories. J Cogn Neurosci 29:1415-1432.

Limousin P, Krack P, Pollak P, Benazzouz A, Ardouin C, Hoffmann D, Benabid AL (1998) Electrical stimulation of the subthalamic nucleus in advanced Parkinson's disease. N Engl J Med 339:1105-1111.

Lin JJ, Rugg MD, Das S, Stein J, Rizzuto DS, Kahana MJ, Lega BC (2017) Theta band power increases in the posterior hippocampus predict successful episodic memory encoding in humans. Hippocampus 27:10401053

Long NM, Burke JF, Kahana MJ (2014) Subsequent memory effect in intracranial and scalp EEG. Neuroimage 84:488-494.

Maddock RJ (1999) Retrosplenial cortex and emotion: new insights from functional imaging studies of the human brain. Trends Neurosci 22:310 316.

Maddock RJ, Garrett AS, Buonocore MH (2001) Remembering familiar people: the posterior cingulate cortex and autobiographical memory retrieval. Neuroscience 104:667-676.

Matsumoto R, Nair DR, LaPresto E, Najm I, Bingaman W, Shibasaki H, Lüders HO (2004) Functional connectivity in the human language system: a cortico-cortical evoked potential study. Brain 127:2316-2330.

Miller SL, Celone K, DePeau K, Diamond E, Dickerson BC, Rentz D, Pihlajamäki M, Sperling RA (2008) Age-related memory impairment associated with loss of parietal deactivation but preserved hippocampal activation. Proc Natl Acad Sci U S A 105:2181-2186.

Minoshima S, Giordani B, Berent S, Frey KA, Foster NL, Kuhl DE (1997) Metabolic reduction in the posterior cingulate cortex in very early Alzheimer's disease. Ann Neurol 42:85-94.

Murdock BB (1962) The serial position effect of free recall. J Exp Psychol 64:482-488.

Nir Y, Mukamel R, Dinstein I, Privman E, Harel M, Fisch L, Gelbard-Sagiv H, Kipervasser S, Andelman F, Neufeld MY, Kramer U, Arieli A, Fried I, Malach R (2008) Interhemispheric correlations of slow spontaneous neuronal fluctuations revealed in human sensory cortex. Nat Neurosci 11:1100-1108. 
Otten LJ, Rugg MD (2001) When more means less: neural activity related to unsuccessful memory encoding. Curr Biol 11:1528-1530.

Paller KA, Wagner AD (2002) Observing the transformation of experience into memory. Trends Cogn Sci 6:93-102.

Palva JM, Palva S, Kaila K (2005) Phase synchrony among neuronal oscillations in the human cortex. J Neurosci 25:3962-3972.

Papma JM, Smits M, de Groot M, Mattace Raso FU, van der Lugt A, Vrooman HA, Niessen WJ, Koudstaal PJ, van Swieten JC, van der Veen FM, Prins ND (2017) The effect of hippocampal function, volume and connectivity on posterior cingulate cortex functioning during episodic memory fMRI in mild cognitive impairment. Eur Radiol 27:3716-3724.

Perlmutter JS, Mink JW (2006) Deep brain stimulation. Annu Rev Neurosci 29:229-257.

Polyn SM, Norman KA, Kahana MJ (2009a) A context maintenance and retrieval model of organizational processes in free recall. Psychol Rev 116:129-156.

Polyn SM, Norman KA, Kahana MJ (2009b) Task context and organization in free recall. Neuropsychologia 47:2158-2163.

Ranganath C, Heller A, Cohen MX, Brozinsky CJ, Rissman J (2005) Functional connectivity with the hippocampus during successful memory formation. Hippocampus 15:997-1005.

Ressler KJ, Mayberg HS (2007) Targeting abnormal neural circuits in mood and anxiety disorders: from the laboratory to the clinic. Nat Neurosci 10:1116-1124.

Rosin B, Slovik M, Mitelman R, Rivlin-Etzion M, Haber SN, Israel Z, Vaadia E, Bergman H (2011) Closed-loop deep brain stimulation is superior in ameliorating parkinsonism. Neuron 72:370-384.

Rugg MD, Vilberg KL (2013) Brain networks underlying episodic memory retrieval. Curr Opin Neurobiol 23:255-260.
Sederberg PB, Gauthier LV, Terushkin V, Miller JF, Barnathan JA, Kahana MJ (2006) Oscillatory correlates of the primacy effect in episodic memory. Neuroimage 32:1422-1431.

Sederberg PB, Kahana MJ, Howard MW, Donner EJ, Madsen JR (2003) Theta and gamma oscillations during encoding predict subsequent recall. J Neurosci 23:10809-10814.

Sestieri C, Corbetta M, Romani GL, Shulman GL (2011) Episodic memory retrieval, parietal cortex, and the default mode network: functional and topographic analyses. J Neurosci 31:4407-4420.

Solomon EA, Kragel JE, Gross R, Lega B, Sperling MR, Worrell G, Sheth SA, Zaghloul KA, Jobst BC, Stein JM, Das S, Gorniak R, Inman CS, Seger S, Rizzuto DS, Kahana MJ (2018) Medial temporal lobe functional connectivity predicts stimulation-induced theta power. Nat Commun 9:4437.

Suthana N, Haneef Z, Stern J, Mukamel R, Behnke E, Knowlton B, Fried I (2012) Memory enhancement and deep-brain stimulation of the entorhinal area. N Engl J Med 366:502-510.

Suthana N, Fried I (2014) Deep brain stimulation for enhancement of learning and memory. Neuroimage 85:996-1002.

Uncapher MR, Otten LJ, Rugg MD (2006) Episodic encoding is more than the sum of its parts: an fMRI investigation of multifeatural contextual encoding. Neuron 52:547-556.

Wagner AD, Shannon BJ, Kahn I, Buckner RL (2005) Parietal lobe contributions to episodic memory retrieval. Trends Cogn Sci 9:445-453.

Zhou Y, Dougherty JH Jr, Hubner KF, Bai B, Cannon RL, Hutson RK (2008) Abnormal connectivity in the posterior cingulate and hippocampus in early Alzheimer's disease and mild cognitive impairment. Alzheimers Dement 4:265-270. 